\title{
Evolución temporal y características sociodemográficas de migrantes venezolanos atendidos en el servicio odontológico de una universidad pública colombiana
}

\section{Temporal evolution and sociodemographic characteristics of Venezuelan migrants treated in the dental service of a Colombian public university}

Jorge Homero Wilches-Visbal ${ }^{(1)}$, Midian Clara Castillo-Pedraza ${ }^{(D)}$, Diana Luz Escobar-Ospino ${ }^{(\mathbb{D} 3}$, Juliana Carolina PalacioBenavides 4

1. Universidad del Magdalena. Santa Marta, Colombia. Fundación Universitaria Los Libertadores. Bogotá, Colombia. Correo: jhwilchev@gmail.com https://orcid.org/0000-0003-3649-5079

2. Universidad del Magdalena. Santa Marta, Colombia. Fundación Universitaria Los Libertadores. Bogotá, Colombia. Correo: midianclar@gmail.com https://orcid.org/0000-0003-3170-3959

3. Universidad del Magdalena. Santa Marta, Colombia. Correo: dianaescobarospino@gmail.com - https://orcid.org/0000-0001-8482-1452

4. Universidad del Magdalena. Santa Marta, Colombia. Correo: jcpalaciob1@gmail.com - https://orcid.org/0000-0001-9954-6668

Tipología: Artículo de investigación científica y tecnológica

Para citar este artículo: Wilches-Visbal JH, Castillo-Pedraza MC, Escobar-Ospino DL, Palacio-Benavides JC. Evolución temporal y características sociodemográficas de migrantes venezolanos atendidos en el servicio odontológico de una universidad pública colombiana. Duazary. 2021 abril; 18(2): 153-162. Doi: https://doi.org/10.21676/2389783X.4078

RESUMEN

Palabras

clave:

migrante;

atención

odontológica;

seguridad

social;

Venezuela;

Santa Marta.

El propósito de este artículo fue analizar la evolución temporal de migrantes venezolanos atendidos en el servicio odontológico de la Universidad del Magdalena, en Santa Marta (Colombia) y examinar algunas de sus características sociodemográficas, entre 2017-II y 2019-I. Para ello, se incluyeron 109 venezolanos encontrados en el archivo de historias del servicio odontológico. Se recolectaron datos sociodemográficos: edad, sexo, estrato socioeconómico, ocupación y estado civil. También se indagó sobre el tipo de afiliación al Sistema General de Seguridad Social en Salud (SGSSS), la empresa prestadora de salud (EPS) y los tratamientos realizados. Se analizaron dos escenarios clínicos: consulta prioritaria y clínica odontológica. Se observó mayor número de mujeres (52\%) que de hombres (48\%), con una media de edad de $27,0 \pm 13,6$ y $29,4 \pm 15,4$ años, respectivamente. Se constató un crecimiento exponencial de venezolanos atendidos entre el 2017-II y el 2019-I. El 45\% de ellos estaba en el SGSSS, y el resto no reportó afiliación. Aproximadamente, el $60 \%$ de los migrantes eran solteros. Las ocupaciones eran variadas; ninguna en el sector formal de la economía. Los tratamientos se concentraron en las áreas de cirugía, odontopediatría y operatoria. Se concluye que los migrantes venezolanos atendidos son en gran parte jóvenes en condición de precariedad económica y sanitaria, que vieron en el servicio odontológico de la universidad una oportunidad para la solución de sus problemas bucodentales.

\section{ABSTRACT}

Keywords:

Migrant; Dental care; Social security; Venezuela; Santa Marta.
The purpose of this study was to analyse the temporal evolution of Venezuelan migrants treated at the dental service of the University of Magdalena-Santa Marta (Colombia) and to examine some of their sociodemographic characteristics, between 2017-II and 2019-I. For this, 109 Venezuelans found in the Archive of Histories of the dental service were included. Sociodemographic data were collected: age, sex, socioeconomic status, occupation, and marital status. The type of affiliation to the General System of

Duazary / ISSN Impreso: 1794-5992 / ISSN Web: 2389-783X / Vol. 18, No. 2 abril - junio de 2021 
Social Security in Health (SGSSS), the Health Provider Company (EPS) and the treatments carried out were also investigated. Two clinical scenarios were analysed: priority consultation and dental clinic (general and specialized consultation). A greater number of women (52\%) than men (48\%) were observed, with a mean age of $27.0 \pm 13.6$ and $29.4 \pm 15.4$ years, respectively. An exponential growth of Venezuelans served between 2017-II and 2019-I was verified. 45\% of Venezuelan patients belonged to the SGSSS and the rest did not report affiliation. Close to $60 \%$ of the migrants were single. Occupations were varied, none in the formal sector of the economy. Treatments were concentrated in areas of surgery, paediatric dentistry, and operaty dentistry. It is concluded that the Venezuelan migrants treated are to a large extent young people in a precarious economic and health condition, who see in the dental service of the university an opportunity for the solution of oral problems.

\section{INTRODUCCIÓN}

Durante los dos primeros siglos de historia republicana, Venezuela se ha caracterizado por un escenario económico fluctuante, que puede dividirse en tres periodos: estancamiento (18201920), crecimiento (1920-1960) y declive (1960$2000)^{1}$. En el periodo de crecimiento, se presentaron diversas oleadas migratorias (muchas ilegales) desde países vecinos y el Caribe $^{2}$.

La migración es un proceso de desplazamiento de individuos entre diferentes territorios que se ha dado desde el inicio de la humanidad, pero no siempre obedeciendo a las mismas circunstancias. A partir de la segunda década del siglo XXI, Venezuela ha enfrentado una crisis económica y humanitaria de enormes proporciones, que ha erosionado la infraestructura sanitaria del país y, por supuesto, la calidad de vida de los ciudadanos ${ }^{3-5}$.

La calidad de vida es un concepto multidimensional que involucra el bienestar económico, de salud y social de un individuo en comunidad. En ese sentido, la calidad de vida está relacionada con oportunidades de empleo, óptimo estado de salud, acceso a vivienda, satisfacción en el lugar de trabajo, seguridad pública, infraestructura urbana, áreas verdes, etc ${ }^{6,7}$.

El detrimento de la calidad de vida en Venezuela tiene origen en el deterioro democrático y la grave crisis de ingobernabilidad derivada de inconsistencias en la política monetaria y fiscal; el declive de la industria petrolera y del valor internacional del petróleo; la destrucción del sistema de precios; la ruptura del estado de derecho; la limitación del pluralismo; el desconocimiento de derechos fundamentales y garantías constitucionales; la criminalización de la oposición, entre otros ${ }^{8}$.

A raíz de lo anterior, han surgido diversas protestas en contra del Gobierno, el cual responde con prácticas que desembocan en abusos contra los derechos humanos de los ciudadanos ${ }^{5,9}$. Como respuesta a la difícil situación social, muchos venezolanos han emigrado forzosamente a diversos países de Latinoamérica y Europa ${ }^{9}$. De acuerdo con datos de la Agencia de la Organización de las Naciones Unidas para los Refugiados (Acnur) y la Organización Internacional para las Migraciones (OIM), el total de venezolanos refugiados $y$ migrantes supera los 4 millones en todo el mundo, en 2019. Los países latinoamericanos son el destino predilecto: para ese mismo año, la OIM y la Acnur estimaron la presencia de 1,3 millones de venezolanos en Colombia, cerca de 768.000 en Perú, y 288.000 en Chile, advirtiendo que las cifras podrían ser superiores ${ }^{10}$.

La migración incontrolada e irregular hacia Colombia, en particular, ha generado impactos socioeconómicos significativos en las regiones receptoras tales como aumento en los sistemas de provisión de servicios, demanda de servicios de educación y salud, dinámicas sociales relacionadas con disputas en el mercado laboral informal, hechos de violencia, etc. De acuerdo con el Banco Mundial, de los 1,3 millones de migrantes contabilizados en Colombia, hasta 2019 , el $77 \%$ son venezolanos, y el $23 \%$ corresponde a colombianos que vivían en el vecino país. Adicionalmente, del $77 \%$ de migrantes de origen venezolano, cerca del $11 \%$ estarían en estatus de irregularidad, lo que representa el factor más agravante para estas personas toda vez que les 
impide acceder a condicionales laborales y sanitarias en igualdad con los nacionales ${ }^{11}$.

El flujo migratorio se concentra fundamentalmente en los departamentos fronterizos (Norte de Santander, Arauca, La Guajira), Bogotá D. C. y la región Caribe ${ }^{12}$. El departamento del Magdalena es el sexto del país en número de migrantes venezolanos, con cerca de $70 \mathrm{mil}$, la mitad de ellos asentados en el distrito de Santa Marta. Cerca de la mitad de los migrantes en el departamento son irregulares, por lo que menos del $20 \%$ de ellos cuenta con seguridad social. A pesar de ello, los entes territoriales han realizado esfuerzos ingentes por brindarles servicios de salud de urgencias, aunque esto repercuta económicamente en el sistema hospitalario departamental por una creciente deuda por concepto de atención ${ }^{12,13}$. Por todo lo reseñado, la migración venezolana es un desafío para los servicios de atención del departamento y la nación.

La atención primaria de salud (APS), entendida como la asistencia sanitaria esencial accesible a todos los individuos a un costo asequible, es el núcleo del sistema de salud de cualquier país y pilar del desarrollo socioeconómico de las poblaciones ${ }^{14}$. Dentro de ella, uno de los servicios básicos es la atención odontológica, dado que la salud bucal incide en la satisfacción de necesidades fisiológicas, psicoafectivas y de aceptación social ${ }^{15}$. Para recibirla, todo extranjero tiene que afiliarse al sistema estatal de salud.

En Colombia, el Sistema General de Seguridad Social en Salud (SGSSS) es la entidad estatal que regula el acceso a servicios de salud, entre ellos los odontológicos. El SGSS atiende a la población a través de tres regímenes: contributivo (destinado a personas con empleo formal o ingresos superiores a un salario mínimo), subsidiado (dirigido a personas sin vínculo laboral vigente o informal, así como aquellas en estado de vulnerabilidad) y de excepción (para militares, policías, docentes del magisterio, entre otros). A diferencia del régimen contributivo, donde existe un pequeño aporte por los servicios, denominado copago, en el régimen subsidiado los servicios de salud son completamente gratuitos ${ }^{16}$. Para recibir atención integral en salud, ciudadanos extranjeros deben estar vinculados a alguno de estos dos regímenes, previo cumplimiento de las exigencias de la autoridad regulatoria de la política migratoria nacional (Migración Colombia); de lo contrario, solo se les garantiza atención de urgencias $^{17}$.

Como la atención odontológica de migrantes venezolanos en entidades públicas universitarias de Santa Marta y el país es poco conocida, el propósito de este trabajo fue examinar algunas características demográficas y la evolución temporal de la cantidad de migrantes venezolanos atendidos en servicio odontólogico de la Universidad del Magdalena, entre 2017-II y 2019-I.

\section{MATERIALES Y MÉTODOS}

\section{Tipo de investigación}

Estudio observacional descriptivo realizado en pacientes migrantes venezolanos atendidos en el servicio odontológico de la Universidad del Magdalena, ubicada en la ciudad caribeña de Santa Marta.

\section{Participantes}

La población objetivo fueron migrantes venezolanos que demandaron servicios de salud oral. Se seleccionaron 109 migrantes de la base de datos perteneciente al archivo de historias de la Clínica Odontológica de la Universidad del Magdalena, la cual posee un formato de registro de ingreso para consulta prioritaria y externa.

Se verificó que los archivos de los pacientes estuvieran debidamente firmados tanto en el formulario de ingreso como en el consentimiento informado voluntario. Se excluyeron aquellos pacientes venezolanos cuyos archivos poseían datos incompletos o con enmendaduras, y tres casos por tener nacionalidad distinta a la venezolana.

\section{Instrumentos}

Se diseñó una matriz en Microsoft Excel para la tabulación de variables sociodemográficas: edad, sexo, estado civil, ocupación, estrato socioeconómico, tipo de régimen del SGSS, empresa prestadora de salud (EPS) y tratamiento recibido, 
con datos provenientes del rango temporal de análisis compuesto por los cuatro periodos comprendidos entre 2017-II y 2019-I.

Para establecer si los pacientes migrantes eran cobijados por el SGSSS, se verificó el tipo y número de documento de identificación, esto es, si contaban con cédula de extranjería (CE), carné diplomático salvoconducto de permanencia (CDSP), pasaporte de la Organización de las Naciones Unidas (PONU) para aquellos en calidad de refugiados o asilados, y permiso especial de permanencia $(P E P)^{17}$. Las variables sociodemográficas fueron analizadas descriptivamente. Para ello, se llevaron a cabo gráficas de caja y bigotes, histogramas, y estimadores centrales y de dispersión.

\section{Procedimiento}

Se analizaron dos escenarios clínicos de atención a la comunidad externa a la institución: consulta prioritaria y clínica odontológica (atendimiento odontológico general y especializado). En estos espacios los estudiantes de pregrado en odontología desarrollan actividades prácticas formativas.

Los migrantes extranjeros en situación no regularizada ante Migración Colombia fueron atendidos en la modalidad de consulta prioritaria a precios módicos, en razón a lo dictaminado en la Resolución 866 de 2017, que solo autoriza la atención de urgencias odontológicas para personas extranjeras en esa condición ${ }^{18}$. Los suscritos a algún régimen del SGSSS podían recibir consulta especializada, además de prioritaria.

\section{Análisis estadístico}

El análisis estadístico se llevó a cabo utilizando el software RStudio versión 1.3.1056, para determinar la asociación entre número de pacientes, estrato socioeconómico, edad y periodo de atención, así como para el resto de los cálculos involucrando las demás variables.

\section{Declaración sobre aspectos éticos}

Para la recolección de los datos se garantizó la confidencialidad y custodia de la información acorde a la Ley Estatutaria 1581 de $2012^{19}$. Se aplicaron los principios y normas éticas de la Declaración de Helsinki ${ }^{20}$. Este estudio, además, presenta ausencia de riesgos según la Resolución 008430 de 1993 del Ministerio de Salud de Colombia ${ }^{21}$. Todas las historias clínicas revisadas contaron con consentimiento informado firmado por cada paciente o su representante legal, quien autorizó que los datos allí consignados pudieran ser utilizados para fines de investigación en cualquier momento.

\section{RESULTADOS}

De los 112 pacientes extranjeros que ingresaron a consulta prioritaria para tratamientos odontológicos de carácter urgente o a la Clínica Odontológica para consulta externa, en el rango de análisis, 109 eran de nacionalidad venezolana, y 3, de otras nacionalidades ( 1 español, 1 argentino y 1 francés). Los venezolanos que llegaron a la consulta fueron seleccionados sin distinción de su situación migratoria. En ese mismo rango, entraron por consulta prioritaria o externa 4649 pacientes colombianos. Con esto, la proporción de migrantes venezolanos atendidos con respecto al total representó el 2,3\%.

De los 109 pacientes venezolanos atendidos, 57 (52\%) fueron mujeres, y 52 (48\%), hombres. Se encontró que el número de venezolanos atendidos por periodo fue de $4(3,7 \%)$ en el 2017-II, $11(10,1 \%)$ en el $2018-I, 31(28,4 \%)$ en el $2018-I I$ y $63(57,8 \%)$ en el 2019-I, como se observa en la Figura 1.

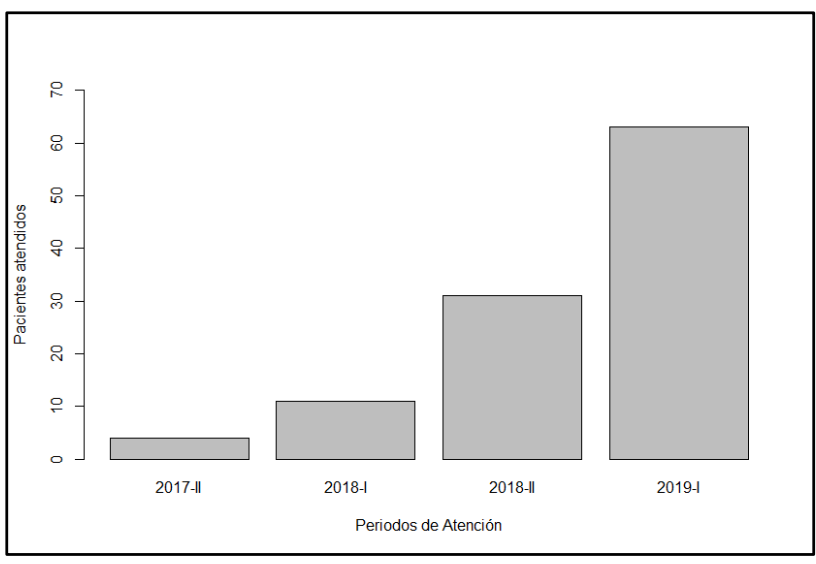

Figura 1. Número de pacientes venezolanos atendidos durante los cuatro periodos de atención en el servicio odontológico de la universidad. 
En la Figura 1 se percibe que la cantidad de pacientes atendidos creció en casi un $1600 \%$, al pasar de 4 migrantes atendidos en 2017-II a 63 en 2019-I. En los dos últimos periodos, se atendieron casi el $90 \%$ de todos los migrantes.

Como la variable edad, en el rango de análisis, se distribuyó normalmente tanto para hombres como para mujeres ( $p>0,05$ en prueba de Lilliefors), se calcularon la media y la desviación estándar (DE) ${ }^{22}$. Así, la media de la edad de las mujeres fue de 27,0 años ( $D E=13,6$ años), mientras que la de los hombres fue de 29,4 años ( $D E=15,4$ años). La cantidad de mujeres superó a la de hombres, en todos los periodos, excepto en 2017-II, cuando igualaron.

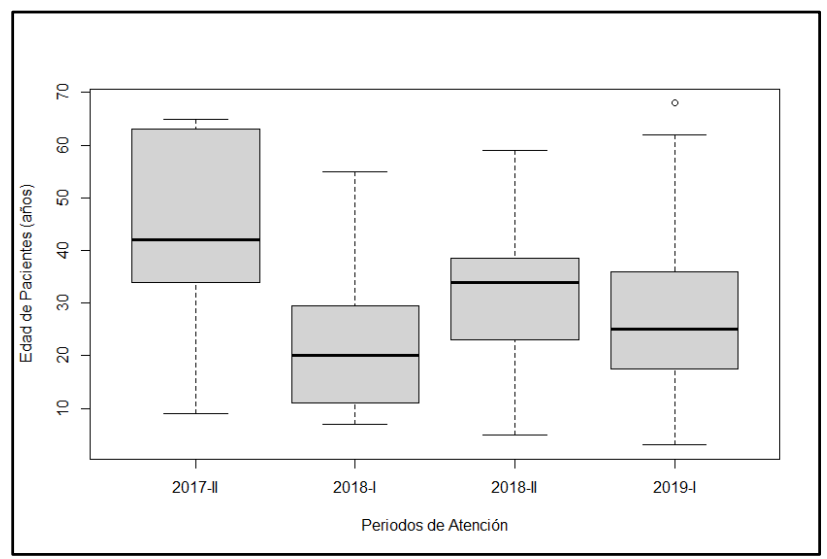

Figura 2. Distribución de pacientes venezolanos atendidos según la edad en los cuatro periodos analizados.

La edad de los pacientes venezolanos por periodo de atención se visualiza en la figura 2 . Se observa que en $2017-$ Il el $75 \%$ de los migrantes se clasificaron entre adultos (36-64 años) y adultos mayores ( $\geq 65$ años), mientras que el $25 \%$ en adultos jóvenes (1835 años), adolescentes (14-17 años) o niños (0-13 años). En 2018-I se percibe una caída drástica en la mediana de la edad de los atendidos (de 42 años a 20 años), y más del $75 \%$ de pacientes registraron edades por debajo de los 36 años. En 2018-II, la mediana subió a 36 años aproximadamente, mostrando una distribución algo simétrica en la cantidad de población joven (1-35 años) y adulta ( $\geq 36$ años). Finalmente, en 2019-I hubo un nuevo descenso en la mediana (de 36 a 25 años). Además, aparece un valor atípico relacionado con un paciente de 68 años. Asimismo, se muestra una tendencia de estabilización de la franja de edad en la población joven ( $<36$ años). También se nota que desde 2018-I hubo un crecimiento sostenido en la atención de niños (descenso gradual del extremo inferior) en los últimos tres periodos. Lo contrario se constata en adultos.

Respecto al estado civil, los migrantes se distribuyeron en $63(57,8 \%)$ solteros, $28(25,7 \%)$ casados y $18(16,5 \%)$ en unión libre. De los solteros, 20 (31,8\%) eran niños; 8 (12,7\%), adolescentes; 19 $(30,1 \%)$, adultos jóvenes; $14(22,2 \%)$, adultos, y 2 $(3,2 \%)$, adultos mayores. Los casados fueron 5 $(17,9 \%)$ adultos jóvenes, $18(64,2 \%)$ adultos y 5 $(17,9 \%)$ adultos mayores. En estado de unión libre hubo $4(22,2 \%)$ adultos jóvenes, $13(72,2 \%)$ adultos y $1(5,6 \%)$ adulto mayor.

Para la variable ocupación, solo se tuvo registro de 71 migrantes. Los migrantes con ocupaciones remuneradas (41) se clasificaron, según el grupo etario, en: 27 (65,8\%) adultos jóvenes, 12 (29,3\%) adultos y $2(4,9 \%)$ adolescentes. Entre ellos, había 7 $(9,9 \%)$ empleadas domésticas, $6 \quad(8,4 \%)$ comerciantes, $4(5,6 \%)$ docentes (no se dieron detalles adicionales), $9 \quad(12,7 \%)$ igualmente distribuidos (3) entre panaderos, conductores y albañiles; 8 (11,3\%), por igual cantidad (2), eran mecánicos, conductores, meseros y niñeras, y 7 $(9,9 \%)$ se desempeñaban en otras actividades. Ninguno manifestó poseer empleo formal. En ocupaciones no remuneradas (30) se encontraban $17(23,9 \%)$ estudiantes (sin especificar el nivel de educación formal), principalmente niños (95\%), y 13 $(18,3 \%)$ amas de casa.

Por otro lado, las intervenciones totales realizadas a los migrantes fueron 333, repartidas en 6 especialidades clínicas. En periodoncia se ejecutaron 36 (10,8\%): 14 (38,9\%) raspados y alisado radicular, $21(58,3 \%)$ profilaxis y 1 (2,8\%) gingivoplastia. En endodoncia, hubo 45 (13,5\%): 18 (40\%) pulpotomías, $14 \quad(31,1 \%)$ endodoncias unirradiculares y $13(28,9 \%)$ multirradiculares. Para rehabilitación oral, solo $2(0,6 \%)$ prótesis fijas. Para operatoria, $59(17,8 \%)$ resinas. En cirugía, 117 $(35,1 \%)$ exodoncias. En odontopediatría, 74 (22,2\%): $33(44,6 \%)$ sellantes, $13(17,6 \%)$ flúor en barniz, 17 $(22,9 \%)$ resinas fluidas y $11(14,9 \%)$ de aparatología. 
En la tabla 1 se evidencia el tipo de vinculación al SGSSS y la EPS a la que pertenecía la población venezolana atendida en cada uno de los cuatro periodos examinados.

Tabla 1. Población venezolana atendida en el servicio odontológico de la Universidad del Magdalena y su vinculación al SGSSS.

\begin{tabular}{|c|c|c|c|}
\hline Periodo & Tipo de régimen & EPS & Población \\
\hline \multirow{2}{*}{ 2017-II } & Subsidiado & Cajacopi & 2 \\
\hline & No reporta & & 4 \\
\hline \multirow{5}{*}{ | I } & \multirow{3}{*}{ Subsidiado } & Salud Total & 1 \\
\hline & & CooSalud & 1 \\
\hline & & Cajacopi & 3 \\
\hline & Contributivo & Nueva EPS & 1 \\
\hline & No reporta & & 5 \\
\hline \multirow{5}{*}{ 2018-II } & \multirow{4}{*}{ Subsidiado } & Nueva EPS & 4 \\
\hline & & Salud total & 3 \\
\hline & & Mutual Ser & 2 \\
\hline & & Cajacopi & 3 \\
\hline & No reporta & & 20 \\
\hline \multirow{5}{*}{ | I-2019 } & \multirow{4}{*}{ Subsidiado } & Mutual Ser & 2 \\
\hline & & Nueva EPS & 12 \\
\hline & & CooSalud & 9 \\
\hline & & Cajacopi & 6 \\
\hline & No reporta & & 31 \\
\hline
\end{tabular}

A partir de la Tabla 1, se deduce que 49 (45\%) pacientes hacían parte del SGSSS, mientras que los $60(55 \%)$ restantes no reportaron afiliación. Así mismo, de los 49 afiliados al SGSSS, solo $1(2 \%)$ era cotizante del régimen contributivo. De los afiliados, el 34,7\% estaba en Nueva EPS; el 28,6\%, en Cajacopi, y el $20,4 \%$ pertenecía a Coosalud, asumiendo en conjunto más del $80 \%$ de las afiliaciones. La primera EPS es de carácter público; las dos últimas, privadas.

Finalmente, en la figura 3 se muestran los pacientes atendidos por estrato socioeconómico durante el rango de análisis. De ella se extrae que el $95 \%$ de los pacientes atendidos eran de los estratos 1,2 y 3 . Entretanto, el $4 \%$ de ellos vivía en zonas de estrato 0 , y $1 \%$, en el 4 . Este último fue el único en el régimen contributivo.

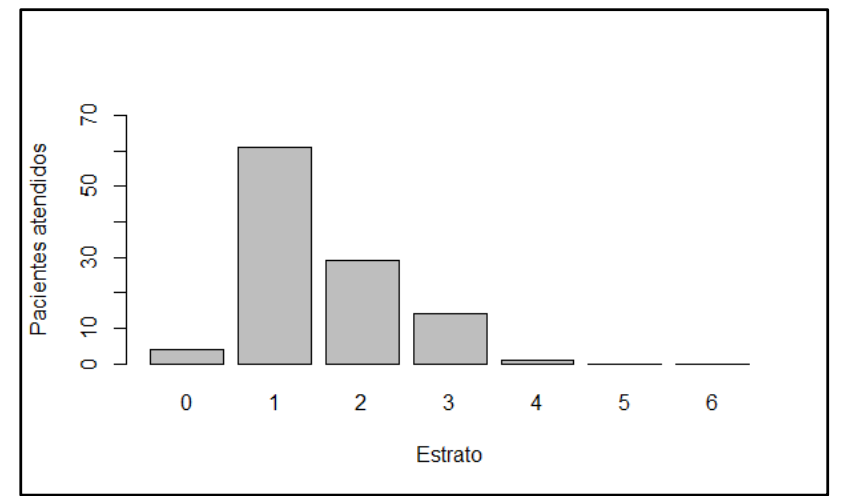

Figura 3. Cantidad de pacientes venezolanos atendidos en los cuatro periodos según el estrato socioeconómico. Rango de análisis: 2017-II-2019-I.

\section{DISCUSIÓN}

La salud bucodental es un aspecto clave para el bienestar general de una sociedad toda vez que alteraciones del sistema estomatognático pueden conducir a cambios significativamente negativos en la integridad física, mental y social de los individuos ${ }^{23}$. Por ello, los Gobiernos nacionales y locales buscan promover hábitos bucales saludables, en la tarea de reducir el impacto de enfermedades prevalentes de este tipo en las poblaciones ${ }^{24}$.

La crisis estructural que vive Venezuela ha ocasionado una diáspora hacia países vecinos, entre ellos Colombia. La condición de vulnerabilidad de los migrantes suele reflejarse en la demanda de servicios de atención primaria como los odontológicos ${ }^{25}$. Bajo esta condición, los servicios públicos de salud oral, en especial los universitarios, se constituyen en el escenario predilecto en los que la población migrante busca dar solución a problemas de salud, entre los que se destacan los bucodentales $^{12,13}$. Por tanto, es válido plantear investigaciones que, como esta, propendan a descubrir cómo ha progresado la necesidad de atención odontológica en estos migrantes desde contextos locales.

De los resultados de este trabajo se extrae que gran parte de los migrantes atendidos son adultos jóvenes y adultos, con tendencia a la baja en la media de edad para los últimos tres periodos. Este es un indicativo de que la población migrante que recibió atención estaba compuesta principalmente 
por jóvenes no mayores a 35 años, concordando con lo reportado por Fernández-Niño et $a{ }^{11}$, quienes en un estudio realizado en Barranquilla (Colombia) encontraron que más del $50 \%$ de migrantes en esa ciudad tenían edades por debajo de los 38 años. Así mismo, en investigaciones realizadas en el Perú se muestra que la mayoría de migrantes venezolanos están entre adolescentes y adultos ${ }^{26,27}$. En este artículo, como en Fernández-Niño et al ${ }^{11}$, Mendoza y Miranda ${ }^{27}$ y Figueroa-Quiñones et $a l^{26}$, la población de mujeres migrantes venezolanas superó a la de hombres, y más de $3 / 4$ partes de las mujeres estaban en edad fértil (14-49 años).

Se constató además un crecimiento exponencial en la cantidad de migrantes atendidos desde el 2017-II, lo cual está en consonancia con el cada vez mayor flujo migratorio hacia ciudades cercanas a las zonas de frontera ${ }^{12}$. Probablemente, el aumento de la población atendida también esté asociado a la percepción positiva de los primeros migrantes respecto a la calidad de la atención recibida en la universidad, al bajo costo de los servicios y a la presencia de docentes especializados que supervisan los procedimientos de los estudiantes. Contrariamente, en los puestos de salud de la ciudad, donde también podían acudir, suele haber escasez de insumos, menor cantidad de profesionales y especialistas, más tiempo de espera y costo en la atención, y riesgo de que ser reportados a Migración Colombia. Ahora bien, debe aclararse que, como los tratamientos ofrecidos en la universidad son de docencia-servicio (para la formación de estudiantes), se requiere que todas las personas paguen una cuota para cubrir los insumos de las intervenciones, sean de consulta prioritaria o general. Con todo, el bajo costo para los migrantes que manifestaban imposibilidad para pagar la cuota se debía a que sus tratamientos eran subsidiados (totalmente o en parte) por la universidad.

Aproximadamente el $60 \%$ de los migrantes eran solteros, y el resto reportó estar casado o en unión libre. La mayoría de solteros eran niños, adolescentes y adultos jóvenes, sumando en conjunto casi el $75 \%$. Los casados se concentraron en la franja etaria de adulto y adulto mayor, con más del $75 \%$. En unión libre, los adultos sumaron más del $70 \%$. La proporción más alta de solteros respecto a casados encontrada en este trabajo concuerda con lo visto en Figueroa-Quiñones et $a l^{26}$, aunque es menor en casados y discrepa, en parte, con lo hallado por Fernández-Niño et $a l^{11}$, donde preponderó la unión libre.

A partir de los datos de ocupación, se pudo establecer que las ocupaciones remuneradas eran acaparadas por migrantes adultos y adultos jóvenes, con poco más del $95 \%$. En las no remuneradas estaban estudiantes, en su mayoría niños, y amas de casa. Ahora, el hecho de que las ocupaciones sean en el sector informal de la economía refleja la precarización laboral de los migrantes, fenómeno confirmado por Fernández-Niño et $a l^{11} \mathrm{y}$ un par de informes recientes elaborados por Revista Semana ${ }^{28}$ y la Universidad Externado de Colombia ${ }^{29}$.

Las intervenciones realizadas superaron al número de migrantes, deduciéndose que hubo cerca de 3 intervenciones por migrante. La exodoncia fue la intervención más recurrente, realizándose en poco más de $1 / 3$ de los pacientes. Le siguieron tratamientos en población pediátrica y en operatoria. Rehabilitación oral fue el área con menor cantidad de intervenciones en migrantes, representando menos del $1 \%$.

Por otra parte, se determinó que alrededor del $84 \%$ de los migrantes pertenecían a los estratos socioeconómicos bajos $(0,1$ y 2$)$. En adición, más del $50 \%$ no contaba con afiliación al SGSSS porque estaba en trámite o porque no habían regularizado su condición migratoria en ese momento. Para estos, la universidad brindó asesoría básica acerca de cómo regularizar su condición ante Migración Colombia.

A partir del análisis de las diferentes variables anteriormente mencionadas, se evidencia, desde un contexto local, el estado de vulnerabilidad de la población migrante venezolana. Esto coincide con lo reportado en otros lugares de Colombia y algunos países de la región ${ }^{25-30}$.

\section{CONCLUSIÓN}

A partir del análisis de características sociodemográficas como edad, sexo, ocupación y estrato, se encontró que más del $50 \%$ de los 
migrantes venezolanos atendidos en el servicio odontológico de la Universidad del Magdalena eran menores de 36 años y tenían una edad media de 26 años, con trabajos en el sector informal de la economía y de estratos bajos. La población de mujeres atendidas fue superior a la de hombres en el rango temporal de análisis. El grueso de las mujeres se encontraba en edad reproductiva.

La cantidad de pacientes atendidos tuvo un crecimiento exponencial del primer periodo de evaluación (2017-II) al último (2019-I). Las áreas odontológicas que asumieron la mayor carga de pacientes fueron cirugía (exodoncias), odontopediatría (sellantes) y operatoria (resinas), con el $75 \%$ del total de intervenciones. Por la condición de precariedad económica manifestada por muchos de ellos, buena parte de los tratamientos fueron subsidiados por la universidad. Menos del $50 \%$ de los migrantes mostraron que estaban vinculados al SGSSS, presuntamente por no haber regularizado su situación ante la autoridad competente. El $95 \%$ de los afiliados era parte del régimen subsidiado, y las EPS con mayor cantidad de migrantes suscritos fueron Nueva EPS, Cajacopi y Coosalud (la primera de estas es pública).

Los docentes y los estudiantes de odontología de la universidad han prestado un servicio de calidad que ha redundado positivamente en el mejoramiento de la calidad de vida de los migrantes a través de las intervenciones terapéuticas relatadas. Para el programa de Odontología, y en especial para sus estudiantes de práctica clínica, el flujo de migrantes venezolanos se constituye en un elemento adicional de entrenamiento clínico.

Finalmente, se necesita una búsqueda más activa de migrantes en condición de irregularidad migratoria por parte de las entidades territoriales de salud, a fin de establecer mecanismos de incorporación efectiva a la sociedad que les permita mejorar su calidad de vida.

\section{DECLARACIÓN SOBRE CONFLICTO DE INTERESES}

Los autores declaran la inexistencia de conflicto de intereses

\section{CONTRIBUCIÓN DE LOS AUTORES}

Primer autor: redacción, diseño metodológico, análisis estadístico y revisión final.

Segundo autor: conceptualización, redacción, diseño metodológico y análisis estadístico.

Tercer autor: recolección de datos y diseño metodológico.

Cuarto autor: recolección de datos y diseño metodológico.

\section{REFERENCIAS BIBLIOGRÁFICAS}

1.Bello OD, Blyde JS, Restuccia D. Venezuela's growth experience. Lat Am J Econ. 2011; 48(2): 199$226 . \quad$ Disponible en: https://scielo.conicyt.cl/scielo.php?script=sci_artte xt\&pid=S0719-04332011000200005.

2.Decorso Sicilia GB. PIB y población desde el periodo tardo colonial hasta 2014: el caso venezolano. Tiempo y Econ. 2017; 5(1): 9-39. Disponible

en: https://ideas.repec.org/a/col/000485/016153.html

3.Page KR, Doocy S, Reyna Ganteaume F, Castro JS, Spiegel $P$, Beyrer $C$. Venezuela's public health crisis: a regional emergency. The Lancet. 2019; 393(10177): 1254-60. Disponible en: https://www.thelancet.com/journals/lancet/article /PIIS01406736(19)30344-7/fulltext.

4.Corrales J. ¿Cómo explicar la crisis económica de Venezuela? Tribuna: Revista de Asuntos Públicos. 2017; (14): 30-4. Disponible en: https://egob.uniandes.edu.co/images/books/tribu na/tb-14.pdf.

5.The Lancet. Venezuelans' right to health crumbles amid political crisis. Lancet. 2019; 393(10177): 1177. Disponible https://pubmed.ncbi.nlm.nih.gov/30910287/.

6.Urzúa M A, Caqueo-Urízar A. Calidad de vida: Una revisión teórica del concepto. Ter Psicológica. 2012; 30(1): 61-71. Disponible en: http://www.scielo.cl/scielo.php?script=sci_arttext 
Jorge Homero Wilches-Visbal, Midian Clara Castillo-Pedraza, Diana Luz Escobar-Ospino, Juliana Carolina Palacio-Benavides

\&pid=S0718-

$48082012000100006 \&$ Ing=en\&nrm=iso\&tlng=en .

7.Velarde-Jurado E, Avila-Figueroa C. Evaluación de la calidad de vida. Salud Pública Méx. 2002; 44(4): 349-61. Disponible en: http://www.scielosp.org/scielo.php?script=sci_artt ext\&pid=S0036-

$36342002000400009 \&$ Ing=es \&nrm=iso\&tlng=es.

8.Rivas Leone JA. Deterioro democrático e ingobernabilidad en Venezuela 1998-2016. Reflexión Política. 2016; 18(36): 158-77. Disponible en:

https://revistas.unab.edu.co/index.php/reflexion/a rticle/view/2659.

9.Alekséenko OA, Pyatakov AN. Venezuela: prueba por la crisis. Inst Latinoamérica Acad Ciencias Rusia. 2019; 2: 57-83. Disponible en: https://www.iberoamericajournal.ru/sites/default/ files/2019/2/alexeenko_pyatakov.pdf.

10.Agencia de la ONU para los Refugiados. Refugiados y migrantes de Venezuela superan los cuatro millones: ACNUR y OIM. 2019 [citado 8 de noviembre de 2020]. Disponible en: https://www.acnur.org/noticias/press/2019/6/5cfa 5eb64/refugiados-y-migrantes-de-venezuelasuperan-los-cuatro-millones-acnur-y.html.

11.Fernández-Niño JA, Vásquez-Rodríguez $A B$, Flórez-García VA, Rojas-Botero ML, Luna-Orozco K, Navarro-Lechuga E, et al. Lifestyles and health status of migrants in a settlement of Barranquilla, Colombia, 2018. Rev Salud Pública. 2018; 20(4): 5308.

12. Banco Mundial. Migración desde Venezuela a Colombia: impactos y estrategia de respuesta en el corto y mediano plazo. Bogotá, Colombia: Banco Mundial; 2018. Disponible en: http://documents1.worldbank.org/curated/en/911 381540835286885/pdf/131472-WP-SPANISHPUBLIC-MigracindesdeVenezuelaaColombia.pdf.

13.Alcaldía Distrital de Santa Marta. Alcaldía Distrital, Gobernación, Gerencia de Fronteras y Gobierno Nacional analizaron la situación migratoria en Santa Marta. 2019 [citado 10 de agosto de 2020].
Disponible

en: https://www.santamarta.gov.co/salaprensa/noticias/alcaldia-distrital-gobernaciongerencia-de-fronteras-y-gobierno-nacional.

14.Organización Mundial de la Salud. Declaración de Alma-Ata. En: Conferencia Internacional sobre Atención Primaria de Salud, Alma-Ata, URSS, 1978. 1978

$$
\text { Disponible }
$$

en: https://www.paho.org/hq/dmdocuments/2012/Al ma-Ata-1978Declaracion.pdf.

15. Díaz Cárdenas S, Tirado Amador LR, Vidal Madera Anaya M. Odontología con enfoque en salud familiar. Rev Cuba Salud Pública. 2014; 40(3): $397-$ $405 . \quad$ Disponible en: http://scielo.sld.cu/scielo.php?script=sci_arttext\&p id=S0864-34662014000300010.

16.Administradora de los Recursos del Sistema General de Seguridad Social en Salud (ADRES). SGSSS en Colombia - ¿Qué es? y ¿Cuál es su Función? 2021 [citado 24 de febrero de 2021]. Disponible en: https://adresfosyga.co/sgsss/.

17. Ministerio de Salud y Protección Social. Afiliación al sistema general de seguridad social en salud de extranjeros y colombianos retornados. 2021 [citado 25 de febrero de 2021]. Disponible en: https://www.minsalud.gov.co/proteccionsocial/Pag inas/afiliacion-al-sistema-de-seguridad-social-ensalud-de-extranjeros-y-colombianos-

retornados.aspx.

18.Colombia. Ministerio de Salud y Protección Social. Decreto 866 de 2017 por el cual se sustituye el Capítulo 6 del Título 2 de la Parte 9 del Libro 2 del Decreto 780 de 2016 Único Reglamentario del Sector Salud y Protección Social en cuanto al giro de recursos para las atenciones iniciales de urgencia prestadas en el territorio colombiano a los nacionales de los países fronterizos. Diario Oficial, 50.244(May. 25 2017). Disponible en: https://www.funcionpublica.gov.co/eva/gestornor mativo/norma.php?i=81836.

19. Colombia. Congreso de la República de Colombia. Ley Estatutaria 1581 de 2012 por la cual se dictan disposiciones generales para la protección de datos personales. Diario Oficial, 48.587 (Oct. 17 2012). 
Evolución temporal y características sociodemográficas de migrantes venezolanos atendidos en el servicio odontológico de una universidad pública colombiana

Disponible en:

http://www.secretariasenado.gov.co/senado/base

doc/ley_1581_2012.html.

20.Asociación Médica Mundial. Declaración de Helsinki de la AMM - Principios éticos para las investigaciones médicas en seres humanos. Helsinky, Finlandia: Asociación Médica Mundial; $1964 . \quad$ Disponible en: https://www.wma.net/es/policies-

post/declaracion-de-helsinki-de-la-amm-principioseticos-para-las-investigaciones-medicas-en-sereshumanos/.

21.Colombia. Ministerio de Salud. Resolución 8430 de 1993 por la cual se establecen las normas científicas, técnicas y administrativas para la investigación en salud. Disponible en: https://www.minsalud.gov.co/sites/rid/Lists/Bibliot ecaDigital/RIDE/DE/DIJ/RESOLUCION-8430-DE1993.PDF.

22. Madadizadeh F, Asar ME, Hosseini M. Common statistical mistakes in descriptive statistics reports of normal and non-normal variables in biomedical sciences research. Iran J Public Health. 2015; 44(11): 1557-8. Disponible en: https://www.ncbi.nlm.nih.gov/pmc/articles/PMC47 03239/.

23.Rosas-Vargas R, De La Teja-Ángeles E, LópezIbarra M, Durán-Gutiérrez $A$. La importancia de la salud bucal reflejada en la salud general de los pacientes pediátricos con trastornos sistémicos. Acta Pediatr Méx. 2015; 36(4): 311-3. Disponible en: http://www.scielo.org.mx/scielo.php?script=sci_art text\&pid=S0186-23912015000400001.

24.Contreras Rengifo A. La promoción de la salud general y la salud oral: una estrategia conjunta. Rev Clínica Periodoncia, Implantol y Rehabil Oral. 2016; 9(2): 193-202. Disponible en: https://www.sciencedirect.com/science/article/pii/ S0718539116300350.

25.Murillo-Pedrozo AM, Agudelo-Suárez AA. Southsouth migration as a social determinant of impact on oral health inequalities and inequities in Latin America. Rev Peru Med Exp Salud Pública. 2019;
36(4): 692-9. Disponible en: http://www.scielo.org.pe/scielo.php?pid=S172646342019000400020\&script=sci_abstract\&tlng=en.

26.Figueroa-Quiñones J, Cjuno J, Ipanaqué-Neyra J, Ipanaqué-Zapata M, Taype-Rondan A. Calidad de vida de migrantes venezolanos en dos ciudades del norte del Perú. Rev Peru Med Exp Salud Pública. 2019; 36(3): $383 . \quad$ Disponible en: https://rpmesp.ins.gob.pe/index.php/rpmesp/articl e/view/4517.

27. Mendoza W, Miranda JJ. La inmigración venezolana en el Perú: desafíos y oportunidades desde la perspectiva de la salud. Rev Peru Med Exp Salud Pública. 2019; 36(3): 497. Disponible en: https://rpmesp.ins.gob.pe/index.php/rpmesp/articl e/view/4729.

28. Rubino AS. Informe del panorama laboral de los venezolanos en Colombia. Bogotá, Colombia; 2020. Disponible

en: https://s3.amazonaws.com/semanaruralvzla/docu mentos/1593761046_informe_mercado_laboralpdf

29. Universidad Externado de Colombia. Panorama laboral de los migrantes venezolanos en Colombia 2014-2019. Bogotá, Colombia; 2020. Disponible en: https://www.uexternado.edu.co/wpcontent/uploads/2020/03/Panorama-laboral-delos-migrantes.pdf.

30.Fernández-Niño JA, Luna-Orozco K. Migración venezolana en Colombia: retos en Salud Pública. Rev la Univ Ind Santander Salud. 2018; 50(1): 5-6. Disponible en: https://revistas.uis.edu.co/index.php/revistasaludu is/article/view/7992 